\title{
Después de un terremoto. Bioética en situaciones de catástrofe
}

\author{
FRANCISCO JAVIER LEÓN C. ${ }^{1, a}$
}

${ }^{1}$ Centro de Bioética. Facultad de Medicina. Pontificia Universidad Católica de Chile. Santiago, Chile.

aDoctor en Filosofía, Magíster en Bioética.

Recibido el 30 de noviembre de 2010 ,

aceptado el 8 de septiembre de 2011.

Correspondencia a Francisco Javier León Correa.

Centro Bioética. Edificio Gastroenterología, $5^{\circ}$ piso. Alameda 340. Santiago. Fax: 6339785 Email: fleonc@uc.cl

\section{Bioethics in catastrophe situations such as earthquakes}

A catastrophe of the magnitude of the earthquake and tsunami that hit Chile not long ago, forces us to raise some questions that we will try to answer from a philosophical, ethical and responsibility viewpoints. An analysis of the basic principles of bioethics is also justified. A natural catastrophe is not, by itself, moral or immoral, fair or unfair. However, its consequences could certainly be regarded as such, depending on whether they could have been prevented or mitigated. We will identify those individuals, who have the ethical responsibility to attend the victims and the ethical principles that must guide the tasks of healthcare and psychological support teams. The minimal indispensable actions to obtain an adequate social and legal protection of vulnerable people, must be defined according to international guidelines. These reflections are intended to improve the responsibility of the State and all the community, to efficiently prevent and repair the material and psychological consequences of such a catastrophe.

(Rev Med Chile 2012; 140: 108-112).

Key words: Earthquakes; Bioethics; Geological Processes; Tsunamis.

\section{Introducción: Después del terremoto}

"F Dueron tres minutos que remecieron gran parte de Chile y de nuestro sistema público de salud. Y si bien los equipos humanos resistieron con vocación y valentía la enorme presión asistencial que se generó, no sucedió lo mismo con nuestros hospitales. Se desplomaron 17 recintos en el centro y sur del país reduciendo, de manera importante, la capacidad de atención y acrecentando la brecha de camas en más de siete mil"1.

Estas palabras de un Informe elaborado poco después del terremoto y posterior maremoto sufridos por Chile, entre el Colegio Médico y el Ministerio de Salud, exponen el desafío que supone una catástrofe natural, con deberes de atención urgentes e impostergables, que tienen que ponerse en marcha sin descuidar la atención general y necesidades de la población. Sin embargo, analizado unos meses después, parece que no resultó tan difícil afrontar la emergencia. La reacción fue rápida.

Como se comentó en editorial de la Revista Médica de Chile poco después: "Ahora, como profesionales de la Salud, debemos afrontar una etapa delicada: la de los efectos psicológicos a mediano y largo plazo originados por el estrés post traumático y los riesgos de salud física derivados de las condiciones sanitarias alteradas por el sismo. Pero podemos contar con la solidaridad, el altruismo, la voluntad y la experiencia de muchos ciudadanos para lograr que Chile se ponga nuevamente de pie"2. Estos valores fueron los que aceleraron la recuperación después del desastre. Podemos verlos también en las experiencias directas narradas por algunos profesionales de la salud que estuvieron presentes ${ }^{3}$.

Una emergencia de esta magnitud nos obliga a plantearnos algunas preguntas, que podemos intentar responder desde la reflexión ética sobre la responsabilidad, y desde el análisis mediante los principios fundamentales de la bioética. 


\section{1. ¿Quién es responsable de atender a los damnificados?}

En un informe, sobre Intervención y Soberanía Estatal ${ }^{4}$ en el marco de las Naciones Unidas, para marcar los límites en intervenciones humanitarias, se reformula el problema desde la definición del "derecho de intervención" a un nuevo concepto de "responsabilidad de proteger", que incluye tres aspectos: responsabilidad de prevenir, de reaccionar y de reconstruir. Aunque no es doctrina general, especialmente en sus consecuencias en el ámbito jurídico, sí sería lo que algunos autores denominan como "el nuevo límite de la soberanía nacional"s.

Prevenir es tarea fundamental del Estado y de cada ciudadano. Reaccionar y reconstruir también son responsabilidad y deber ético del Estado, y de todos. Pero hay que delimitar bien quienes tienen ese deber, cuando se producen catástrofes naturales. La pregunta sería: ¿quién es responsable por la reparación de daños materiales o morales en situaciones -como la del terremoto- en las que ningún autor puede ser inculpado de haberlas causado? Podríamos delimitar, en un primer momento, que el estado tiene deberes de justicia por el principio de subsidiariedad, mientras los grupos sociales y los individuos tienen deberes éticos de solidaridad, no de estricta justicia: "Individuos responsables deben formar grupos capaces de asumir en forma efectiva la responsabilidad por la eliminación de daños morales, incluso aunque ellos no puedan ser culpabilizados por la existencia de esos daños morales" ${ }^{\text {. }}$. No todos pueden ayudar en todo, pero se pueden delimitar las acciones de cada individuo dentro del grupo o de los grupos dentro de las comunidades más amplias.

Es cierto que no puede considerarse una catástrofe natural en sí misma como moral o inmoral, justa o injusta; sin embargo, sus consecuencias sí podríamos tratarlas de esta manera, dependiendo de si hubiesen podido ser evitadas o disminuidas. Las personas no son responsables en primer grado por los golpes del destino, pero sí lo son en un grado secundario, en la medida en que su acontecer o sus efectos pueden ser evitados o disminuidos. Pero las responsabilidades del cargo que ocupa un agente sí pueden suponer un grado mayor de exigencia ${ }^{7}$, muy especialmente de los que desempeñan cargos públicos, que tienen el deber ético de preocuparse por el bien público y por esa tarea de prevención, reacción oportuna y acciones de reconstrucción.

Los principios de justicia se refieren al orden institucional, mientras los principios morales más amplios regulan la relación entre los individuos: "uno tiene la obligación de ayudar a personas que incluso no tienen ninguna relación con nuestro orden institucional". Así, por ejemplo, en catástrofes naturales, estamos siempre obligados a ayudar no por criterios éticos de justicia, pero sí de solidaridad.

\section{Principios éticos de actuación médica ante un desastre natural}

"Un desastre es un acontecimiento grave o repentino de tal magnitud que la comunidad afectada necesita esfuerzos extraordinarios para afrontarlo, a menudo con apoyo externo o ayuda internacional" 9 . Para los profesionales de la salud, "estas situaciones van acompañadas de un desequilibrio fuerte e imprevisto entre las posibilidades de intervención de los médicos y las necesidades de las víctimas o personas cuya salud está amenazada, en un tiempo dado" 10 .

Las catástrofes naturales suponen un contexto peculiar para el debate en bioética, con conflictos y dilemas entre valores y principios éticos que se plantean de forma diferente a la atención de salud ordinaria: tienen siempre un gran impacto en la salud pública; hacen que se replantee la distribución de recursos sanitarios; cuestionan las políticas actuales en materia de educación sanitaria, prevención y gestión de emergencias; cuestionan las prioridades; precisan enfoques amplio e interdisciplinares; revelan la importancia de la cooperación mundial en la investigación y obtención de datos fiables.

Un primer aspecto, al igual que en la ética clínica, es no dañar más, no aumentar el daño ya causado, la no maleficencia. Las catástrofes inician una reacción multidisciplinaria que pone en marcha numerosos tipos de ayuda, que van desde los transportes y aprovisionamiento de alimentos hasta la atención médica, pero siempre dentro de un marco de seguridad. Estas operaciones necesitan una autoridad eficaz y centralizada que coordine las acciones públicas y privadas.

Los escasos recursos médicos y/o la desorganización en terreno y el aumento de heridos en poco 
tiempo, crean un verdadero problema ético -según la Asociación Médica Mundial (AMM)-, sobre todo en "la selección de las medidas médicas y las prioridades en el tratamiento y administración basados en un diagnóstico rápido y un pronóstico para cada paciente ${ }^{10}$.

También es un delicado problema de justicia, en el primer momento de la atención, la selección de las víctimas según su situación clínica de mayor o menor gravedad. Es uno de los principales retos éticos para el médico, que debe afrontar las decisiones de "triage" conforme a su "deber de no insistir a cualquier costo y tratar a personas en urgencias sobrepasadas y así desperdiciar recursos escasos necesarios para otras víctimas. La decisión de no tratar a una persona herida, por las prioridades que establece la catástrofe, no puede ser considerada como una falta en la ayuda a una persona en peligro de muerte. Es justificado cuando se intenta salvar la máxima cantidad de personas. Sin embargo, el médico debe tener compasión por esos pacientes, ayudarlos y respetar su dignidad, por ejemplo, aislándolos y dándoles calmantes apropiados"10.

Es fundamental desde el análisis ético que la intención del médico sea salvar la mayor cantidad de vidas humanas, y en lo posible realizar cuidados paliativos con los que ya no pueden restablecerse. En ningún momento de esta Declaración de la AMM se justifica la intervención del médico para acelerar la muerte de esos pacientes ya irrecuperables por compasión, que sería eutanasia, sino únicamente ayudarlos, aislarlos, y realizar sedación terminal si es preciso, para ayudarles a afrontar la muerte sin dolor, sin intenciones eutanásicas: "La eutanasia, es decir, el acto deliberado de poner fin a la vida de un paciente, aunque sea por voluntad propia o a petición de sus familiares, es contraria a la ética", según Declaración de la misma AMM en 2001, en que "reafirma su firme convicción de que la eutanasia entra en conflicto con los principios éticos básicos de la práctica médica, (e) insta resueltamente a todas las asociaciones médicas nacionales y a los médicos a abstenerse de participar en la eutanasia, incluso si está permitida o despenalizada por la legislación nacional, bajo ciertas condiciones ${ }^{11}$.

En una crisis sanitaria hay que realizar un trabajo técnico impecable; hay que dedicar mucho tiempo y esfuerzo al trabajo con los medios de comunicación; y, lo que se suele llevar peor, hay que afrontar la utilización política de las crisis ${ }^{12}$. Desde la bioética, correspondería a la aplicación del principio de justicia ${ }^{13}$ : investigar para mejorar el conocimiento de las catástrofes y sus efectos en la salud pública; adoptar una actitud y política proactiva (sistemas de alerta y monitorización); priorizar las políticas de las medidas de prevención; establecer un uso crítico del principio de precaución en la evaluación de riesgos; potenciar la educación ambiental; la participación política de todos en las soluciones y la rendición de cuentas de lo realizado; y finalmente, promover las iniciativas solidarias y la labor de las instituciones de cooperación mundial en políticas de sostenibilidad.

Sabemos que el principio de autonomía cede en situaciones de urgencia ante la necesidad imperiosa de implementar acciones que salvaguarden la vida del paciente. Pero enseguida hay que considerar el deber ético -ligado al respeto por la autonomía del paciente- de promover como profesionales de la salud su competencia, y en el caso de catástrofes, la participación de las personas y poblaciones afectadas lo más posible, de forma que promovamos su autonomía y movilicemos los recursos positivos que tienen ${ }^{14}$.

Es fundamental finalmente la solidaridad ${ }^{15}$. Es parte del principio más general de beneficencia, e imprescindible complemento de la justicia en el actuar moral ${ }^{16}$. Aunque lo indicamos en el último lugar, es precisamente lo que motiva la respuesta moral. La compasión por el desastre que han sufrido otros fundamenta nuestra respuesta ética ${ }^{17}$, nos pone en el lugar del otro, nos mueve a compasión, pero además nos interpela íntimamente porque nos construimos moralmente en la interrelación con los demás ${ }^{18}$, y el rostro de los que sufren exige de nosotros una respuesta de cuidado del otro ${ }^{19}$.

\section{Respuestas mínimas imprescindibles}

Recogemos ahora aspectos desarrollados en Guías de acción de salud ante emergencias humanitarias y situaciones de catástrofe ${ }^{20}$, que pretenden exponer un conjunto de respuestas mínimas para proteger y mejorar la salud y el bienestar psicosocial de las personas afectadas y sentar así las bases de tareas ulteriores más integrales y el inicio de la reconstrucción, una vez estabilizada la situación.

Los principios éticos contenidos en varias Guías internacionales ${ }^{21-23}$, son los siguientes: 


\section{a. Promover los derechos humanos y la equidad}

Quienes proporcionan asistencia humanitaria deberían promover los derechos humanos de todas las personas afectadas y proteger a los individuos y a los grupos que corren mayor riesgo de conculcación; además, deberían promover la equidad y la no discriminación, sean cuales fueren el género, el grupo de edades, los idiomas, el grupo étnico y la ubicación.

\section{b. Participación de las poblaciones afectadas: promover la competencia y autonomía}

Se debe maximizar la participación de las poblaciones afectadas en la respuesta de asistencia humanitaria, posibilitando que los diferentes grupos locales recuperen o retengan el control sobre todas las decisiones que afectan a sus vidas, y que establezcan un sentido de apropiación local imprescindible para lograr la calidad, equidad y sostenibilidad de los programas.

\section{c. Ante todo, no causar daños no intencionales}

Ya lo mencionamos anteriormente.

\section{d. Aprovechar y maximizar los recursos y capacidades disponibles}

Un principio fundamental -incluso en las etapas iniciales de una emergencia- es el fomento de las capacidades locales, apoyando la autoorganización y la autoayuda y fortaleciendo los recursos ya existentes. Con frecuencia, los programas impulsados y ejecutados sólo desde el exterior conducen a soluciones inapropiadas con escasa sostenibilidad $^{21}$.

\section{e. Sistemas de apoyo integrados}

En búsqueda de la mayor beneficencia. La proliferación de servicios y los servicios autónomos, pueden crear un sistema de atención sumamente fragmentado. Las actividades integradas en sistemas más amplios tienden a beneficiar a mayor cantidad de personas y suelen ser más sostenibles ${ }^{23}$.

\section{La protección social y jurídica de los damnificados}

Es preciso detectar, vigilar, prevenir y responder a las amenazas a la protección y las deficiencias en los sistemas de resguardo, y responder a dichas amenazas, mediante medidas de protección social de los derechos humanos fundamentales ${ }^{20}$.

Esos problemas de protección no sólo redundan en sufrimientos inmediatos, sino que también pueden perturbar la reconstitución de las redes sociales con un sentido de comunidad, factores que tienden a ser de importancia crítica para apoyar el bienestar psicosocial. Es fundamental para el impacto psicológico en desastres "el nivel de percepción de amenaza vital, el nivel de apoyo social después del trauma, el tipo de respuesta emocional peri-traumática y el nivel de disociación peri-traumática"24.

La protección social se logra activando y fortaleciendo las redes sociales y los mecanismos comunitarios que reducen los riesgos y satisfacen necesidades inmediatas ${ }^{25}$. La protección es una responsabilidad colectiva de los Estados, las poblaciones afectadas y quienes prestan asistencia humanitaria. En gran parte se logra cuando los residentes locales se organizan entre sí para responder a las amenazas, y por ende, cobran conciencia de que pueden valerse por sí mismos y pueden contar con mecanismos sostenibles de defensa de sus derechos ${ }^{26}$.

Después de la respuesta inmediata, es preciso tomar medidas de protección jurídica que aseguren la continuidad de la protección ${ }^{20}$. El desmoronamiento de la ley y del orden público que ocurre en muchas crisis acrecienta la vulnerabilidad de la gente a las violaciones a sus derechos y a las medidas de salvaguarda que ofrecen los sistemas jurídicos internacionales y nacionales ${ }^{27}$. La protección jurídica dimana de la aplicación del derecho internacional humanitario y la legislación internacional sobre derechos humanos, que consagran los derechos intrínsecos de todas las personas, con medidas especiales de protección para grupos en situación de riesgo ${ }^{28-31}$. Los Estados son primordialmente responsables de proteger a los residentes en sus territorios

La seguridad, la dignidad y la integridad son conceptos fundamentales en el derecho humanitario internacional y también son fundamentales para el enfoque médico y la acción humanitaria. La protección jurídica promueve la dignidad, la valía personal y la seguridad, y fortalece la responsabilidad social.

Son reflexiones desde la bioética que se pretende sirvan para mejorar la responsabilidad -no sólo del Estado sino de todos- de prevenir mejor, 
reaccionar con más rapidez, y reconstruir de modo más efectivo, no sólo en lo material, sino en la recuperación psicosocial de los afectados.

\section{Referencias}

1. Rodríguez Whipple P. Editorial. Revista Vida Médica 2010; 62 (1).

2. Reyes H. Chile se pone de pie otra vez. Editorial. Rev Med Chile 2010; 138: 267-9.

3. O’Ryan M. Reflexiones y sentimientos desde un equipo de voluntarios del Ministerio de Salud y el Colegio Médico de Chile, después del terremoto reciente. Rev Med Chile 2010; 138: 270-3.

4. ICISS. Comisión Internacional sobre Intervención y Soberanía Estatal. The Responsibility to Protect. Report of the International Commission on Intervention and State Sovereignty. http://www.iciss.ca/report-en.asp (revisado 18 julio 2011).

5. Ferrada A. La fuerza del ejemplo. Exploraciones del paradigma del juicio. Barcelona: Gedisa; 2008.

6. Gosepath S. Responsabilidad por la eliminación de daños morales. En: Cortés F, Giusti M (Editores) Justicia global, derechos humanos y responsabilidad. Bogotá: Siglo del Hombre; 2007.

7. Platts M. Introducción: responsabilidades. En: Hansberg O, Platts M (Compiladores) Responsabilidad y libertad. México: UNAM y FCE; 2002 p. 13-33.

8. Kreide R. Justicia global, pobreza y responsabilidad: ¿tienen obligaciones las empresas transnacionales? En: Cortés F, Giusti M. ob.cit.: 101-33.

9. Noji EK. The Public Health Consequences of Disaster. Oxford: University Press; 1997.

10. Asociación Médica Mundial. Declaración sobre la Ética Médica en Casos de Catástrofes. 46 Asamblea General, Estocolmo; 1994. y revisada por la $57^{\mathrm{a}}$ Asamblea General de la AMM, Pilanesberg, Sudáfrica, octubre 2006.

11. Asociación Médica Mundial. Declaración sobre la eutanasia. Adoptada por la 38 asamblea Médica Mundial, Madrid, España, octubre de 1987, revisada y adoptada por la 53 $3^{\mathrm{a}}$ Asamblea General de la AMM, Washington 2002 May 2001 20.3/2001.

12. El Sistema Sanitario ante situaciones de crisis. Madrid: Fundación de Ciencias de la Salud; 2008.

13. Moreno M. Modulaciones del principio de justicia en medicina de catástrofes y ética ambiental. En: VII Congreso Nacional de Bioética. Valencia: Asociación de
Bioética Fundamental y Clínica, Fundación Étnor; 2005.

14. Alfonso-Galán MT, Hornos F, Piga A. La ética de la preparación de comunidades locales para afrontar situaciones de catástrofes. Revista de Bioética Latinoamericana, Venezuela; 2010; 5: 5.

15. De Velasco JM. La bioética y el principio de solidaridad. Bilbao: Universidad de Deusto; 2003.

16. Cortina A. Alianza y contrato. En: Política, ética y religión. Madrid: Editorial Trotta, 2a ed.; 2005: 155-6.

17. Nancy JL. Ser singular plural. Nosotros. Madrid: Arena Libros; 2006.

18. Ricoeur P. Sí mismo como otro. Madrid: Siglo XXI; 1996.

19. Levinas E. Entre nosotros. Ensayos para pensar en otro. Valencia: Pre-Textos; 1993.

20. IASC. Guía del Inter-Agency Standing Committee sobre Salud Mental y Apoyo Psicosocial en Emergencias Humanitarias y Catástrofes. Ginebra: IASC; 2007.

21. Sphere Project. Humanitarian Charter and Minimum Standards in Disaster Response, 2004, págs. 51-102. Ginebra: Sphere Project. http://www.sphereproject.org/ handbook/index.htm (revisado 18 julio 2011)

22. IASC. Directrices para intervenciones sobre VIH/sida en contextos de emergencia. Ginebra; 2003.

23. IASC. Directrices aplicables a las Intervenciones contra la Violencia por Razón de Género en Situaciones Humanitarias. Ginebra. IASC; 2005.

24. Figueroa R, Marín H, González M. Apoyo psicológico en desastres: Propuesta de un modelo de atención basado en revisiones sistemáticas y metaanálisis. Rev Med Chile 2010; 138: 143-51.

25. Slim H, Bonwick A. Protección: Una guía de ALNAP para las agencias humanitarias. 2005.

26. ALNAP. Manual para la participación de poblaciones afectadas por la crisis en la acción humanitaria. 2003. http://www.alnap.org/publications/gs_handbook/gs_ handbook.pdf y http://www.psicosocial.net (revisados 18 julio 2011)

27. ACTION AID. Learning About Rights. Module three: law and rights in emergencies. 2001. http://www.reliefweb. int/library/library/actionaid-rights-2001.html (revisado 18 julio 2011).

28. ACNUR. Manual para situaciones de emergencia. 2002.

29. Amnesty International. http://www.amnistiainternacional.org/ (revisado 18 julio 2011).

30. Human Rights Watch. http://www.hrw.org/spanish/ (revisado 18 julio 2011).

31. Comisión Interamericana de derechos humanos. http:// www.cidh.org/Default.htm (revisado 18 julio 2011). 